\title{
Understanding Interactions Between Caregivers and Care Recipients in Person-Centered Dementia Care: A Rapid Review
}

This article was published in the following Dove Press journal: Clinical Interventions in Aging

\author{
Qiujuan $\mathrm{Wu}^{\mathrm{l}, *}$ \\ Siyu Qian (D) $^{2,3, *}$ \\ Chao Deng $\mathbb{D}^{4,5}$ \\ Ping $\mathrm{Yu}$ (D) $^{2,4}$
}

'Oncology Department, The Fifth Affiliated Hospital of Guangzhou Medical University, Guangzhou, Guangdong Province, People's Republic of China; ${ }^{2}$ Centre for Digital Transformation, School of Computing and Information Technology, Faculty of Engineering and Information Sciences, University of Wollongong, Wollongong, NSW 2522, Australia; ${ }^{3}$ Drug and Alcohol Service, Illawarra Shoalhaven Local Health District, Wollongong, NSW, 2500, Australia; ${ }^{4}$ Illawarra Health and Medical Research Institute, Wollongong, NSW 2500, Australia; ${ }^{5}$ School of Medicine, Faculty of Science, Medicine and Health, University of Wollongong, Wollongong, NSW, 2522, Australia

*These authors contributed equally to this work
Correspondence: Ping Yu

Centre for Digital Transformation, School of Computing and Information

Technology, Faculty of Engineering and Information Sciences, University of

Wollongong, Wollongong, New South

Wales 2522, Australia

Tel +6I 242215412

Fax +6I 2 422I 4045

Email ping@uow.edu.au
Background: Good interactions are essential in caring for people with dementia. There is a lack of knowledge about interaction approaches used by caregivers in personcentered dementia care. This study aimed to understand interactions in person-centered dementia care.

Methods: A search for relevant publications was undertaken in 2020 on two electronic databases, MEDLINE with full text and CINAHL Plus with full text. This was supplemented by manual searching of the reference lists of relevant articles. Inclusion and exclusion criteria were applied to determine the relevance of the articles. Data extraction included publication year, country, study setting, aim, design, definition of person-centered dementia care, elements of person-centered dementia care and interaction approaches used by caregivers. A Donabedian quality framework was used to group the elements of person-centered dementia care into three categories: structure, process and outcome.

Results: A total of 25 articles were included in the review, all from developed countries. A conceptual framework was developed for the delivery of person-centered dementia care. It includes the organizational structure, ie, management and resources, a competent workforce and physical environment; the dementia care process, ie, respectful interaction underpinned by good knowledge about the care recipients in a calm, peaceful environment; and care outcome, ie, the social, psychological, and physical well-being of the care recipients. Interaction approaches used by caregivers in providing person-centered dementia care were classified according to the six purposes of interaction: to know and understand the care recipient, to keep the person happy and satisfied, to make the person feel safe and secure, to calm the person, to support self-identity, and to guide the person in conducting daily activities.

Conclusion: The delivery of person-centered dementia care needs to consider organizational structure, the dementia care process, and care outcome which together foster a positive environment for meaningful interactions between caregivers and care recipients. The identified interaction approaches could be used by dementia care trainers to develop training materials.

Keywords: dementia, framework, interaction, person-centered care

\section{Plain Language Summary}

What is known on the subject?

- Person-centered dementia care has gained considerable attention, but there remains limited knowledge about care interaction approaches in delivering this care.

- Good interactions are essential in caring for people with dementia. 


\section{What this paper adds to existing knowledge?}

- It identified elements of person-centered dementia care.

- It also identified interaction approaches in providing person-centered dementia care.

\section{What are the implications for practice?}

- The delivery of person-centered dementia care needs to consider organizational structure, dementia care process, and care outcome to foster a positive care environment.

- Dementia care trainers could use the identified interaction approaches to develop training materials.

\section{Introduction}

With population aging, the number of people living with dementia is rapidly increasing. In 2019, the Alzheimer's Disease International estimated that over 50 million people worldwide had dementia, and there will be 152 million people living with dementia by $2050 .{ }^{1}$ There is an urgent need to improve quality of care for people with dementia. ${ }^{2}$

Developed by Professor Thomas Kitwood in the 1980s, person-centered dementia care has gained considerable attention. $^{3-5}$ Despite the frequent use of this term in the literature, the concept of person-centered dementia care is not a straightforward one. ${ }^{4}$ Kitwood's conceptualization focused on preserving the personhood of people with dementia. ${ }^{6}$ Using Kitwood's work as the backbone, Brooker theorized four major components of person-centered dementia care (VIPS model): (1) valuing people with dementia and those who care for them, (2) treating people as individuals, (3) looking at the world from the perspective of the person with dementia, and (4) a positive social environment in which the person living with dementia care experience relative well-being. ${ }^{4,7}$ Through a literature review, Edvardsson et al identified six components of person-centered care for people with severe Alzheimer's disease. ${ }^{8}$ These components were (1) regarding personhood in people with Alzheimer's disease as increasingly concealed rather than lost, (2) acknowledging the personhood of people with Alzheimer's disease in all aspects of care, (3) personalizing care and surroundings, (4) offering shared decision-making, (5) interpreting behavior from the person's viewpoint, and (6) prioritizing the relationship to the same extent as the care tasks. ${ }^{8}$ Recognizing the need for a single framework for person-centered dementia care in order to make this care practice the norm, Love and Pinkowitz developed a four-component conceptual framework using a consensus-based approach with dementia care experts and literature review. ${ }^{9}$ These components included (1) core values and philosophy, (2) structure, (3) operational practices and (4) individualized practices, ie, individual ways of interacting with people with dementia. ${ }^{9}$

Both Edvardsson et al and Love and Pinkowitz's conceptualization covered elements of relationship or interaction between caregivers and care recipients with dementia. This is due to the fact that, to deliver person-centered dementia care, caregivers need to know about the person through interpersonal relationships. Recognizing the importance of developing good care relationship, researchers studied relationship-centered care. ${ }^{10-12}$ Nolan et al proposed a "Senses Framework" describing six potential dimensions of relationship-centered care, namely security, belonging, continuity, purpose, achievement and significance. ${ }^{10}$ They suggested that to achieve good care, all parties in the care relationship need to experience these senses. ${ }^{10}$ It is through the day-to-day interactions that relationships develop between caregivers and care recipients with dementia.

Both person-centered care and relationship-centered care recognize the importance of interactions between caregivers and care recipients with dementia. Adams and Gardiner reviewed literature and their own clinical practice on communication and interaction between people with dementia, their informal caregivers and formal professional caregivers. ${ }^{13}$ They identified various types of communications that caregivers used to help people with dementia to express thoughts, feelings and wishes. Adams and Gardiner's review only yielded three empirical studies published in or before 2001, there is a need to synthesize findings from recent studies on how caregivers interact with care recipients with dementia according to the principles of person-centered care.

This study aimed to understand the actual interactions between caregivers and care recipients with dementia through a rapid review of literature that reported relevant findings. It first sought to synthesize from these studies, the definitions they used for person-centered dementia care and the elements of person-centered dementia care. This information served as a theoretical base for understanding the context in which the interactions occurred. It then synthesized the interaction approaches used by caregivers when interacting with care recipients with dementia. As relationship is an element of person-centered care, ${ }^{8,9}$ McCormack argues that person-centered care is a more inclusive term than relationship-centered care. ${ }^{14}$ We used the term person-centered care. 


\section{Materials and Methods}

A rapid review methodology ${ }^{15}$ was used to conduct this review. It has been applied in a variety of healthcare areas, including palliative care, ${ }^{16}$ e-mental health ${ }^{17}$ and behavioral risk factors. ${ }^{18}$ This methodology is "a form of evidence synthesis that may provide more timely information for decision-making." ${ }^{19}$ It utilizes similar processes to systematic review but limits the scope (eg, databases) and various aspects of synthesis (eg, data extraction). ${ }^{16,20,21}$

\section{Search Methods}

A search for literature published since 2000 was undertaken in June 2020 on two electronic databases MEDLINE with full text and CINAHL Plus with full text. The search terms "interaction", "person-cent*", "relationship-cent*" and "dementia" were used in combination to identify articles (see Supplementary Table 1). References in the included articles were also screened. The following criteria were used in the selection of articles.

\section{Inclusion Criteria}

Peer-reviewed English publications which report interactions between caregivers (either formal or informal) and care recipients with dementia according to the principles of person-centered care. Hereafter, we use caregivers to collectively refer paid/formal and family/informal caregivers.

\section{Exclusion Criteria}

- Articles not dealing with dementia care.

- Articles not addressing person-centered care.

- Articles not reporting the actual interaction between caregivers and care recipients with dementia.

- Literature review and contextual articles, protocols, instrument development editorials, letters and dissertations.

- Studies of aged care instead of dementia care.

- Summary and analysis of policy, books, duplicated articles on the same study.

- Conference or other non-journal articles.

\section{Search Outcome and Screening Process}

The article search and screening process is presented in Figure 1. The initial search yielded 215 articles. All the articles were exported to Endnote X7 to remove duplicates, leaving 131 articles for title and abstract screen. The title and abstract of each article were copied and pasted to an excel spreadsheet for reading and assessing against the inclusion and exclusion criteria (Supplementary Table 2). This resulted in 46 articles included for full-text screen (Supplementary Table 3). After full-text screen, 20 articles were included. An additional five articles were identified from the reference lists. In total, 25 articles were included in this review (Supplementary Table 4). The first two authors independently screened all the articles, compared their screening results and resolved any disagreement.

\section{Data Extraction and Synthesis}

Data extraction was conducted using Microsoft Excel. Four categories of data were extracted: (1) characteristics of the studies including publication year, country, study setting, aim, and design/method/participant, (2) definition of person-centered dementia care, (3) elements of personcentered dementia care, and (4) descriptions on how caregivers interacted with a care recipient with dementia. To extract these data, each article was read multiple times to highlight relevant information. These data were then copied and pasted into excel spreadsheets, each spreadsheet contained one category of data. The reference from which the data were extracted was also recorded. The first two authors independently extracted data from half of the articles, then cross-checked each other's extraction and discussed and resolved disagreement.

Thematic analysis ${ }^{22}$ was conducted to synthesize findings through an iterative coding, comparison and grouping process. Descriptive themes were developed and analytical themes were then generated from these themes. The elements of person-centered dementia care were organized into the three categories of Donabedian quality framework $^{23}$ - structure, process and outcome. The Donabedian framework is a conceptual model for assessing healthcare quality. This framework has been used to synthesize evidence on person-centered care in residential aged care ${ }^{24}$ implementation of person-centered care, ${ }^{25}$ and quality indicators of palliative care for older people with dementia. ${ }^{26}$ It provides a useful mechanism for analyzing elements of person-centered dementia care and displaying relationships between these elements.

\section{Results}

\section{Characteristics of Studies}

All the studies were conducted in developed countries and almost all of them were conducted in Europe (Supplementary Table 5). Seventeen studies were 


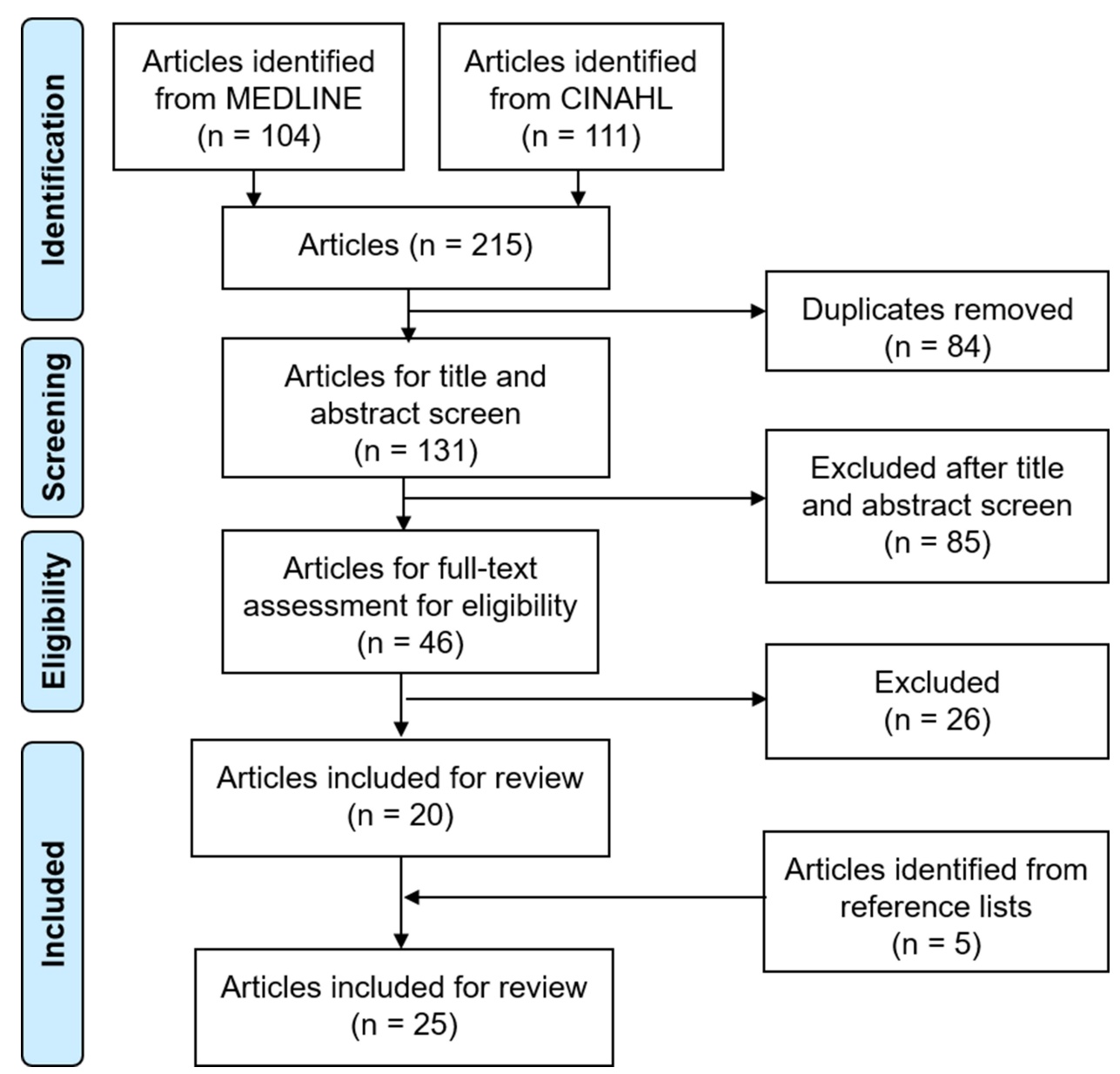

Figure I Article search and screening process.

conducted in residential aged care homes. Twenty-two studies were published in or after 2010. This might be because the research in person-centered dementia care was focused on the development of standards and its application instead of interaction before this time.

\section{What is Person-Centered Dementia Care?}

Fifteen studies discussed the definition of person-centered dementia care according to the authors' own understanding or by reference to other literature. Person-centered dementia care is provided through interaction between caregivers and care recipients. ${ }^{27-30}$ The goal is to maintain care recipients' present abilities, ${ }^{31-33}$ physical and mental health, ${ }^{27,33}$ well-being ${ }^{29,34}$ and dignity. ${ }^{33,35-37}$ To achieve this goal, caregivers need to "see the person behind the disease" 28,37 and acknowledge the personhood of care recipients, ${ }^{29,37-39}$ that is, to understand the care recipients' biography, ${ }^{27,32,40}$ value, ${ }^{29,32}$ personality, ${ }^{27,32}$ and care preferences and needs. ${ }^{36}$ When interacting with care recipients, caregivers need to respect them ${ }^{30,33,35,36,41}$ and build a calming, peaceful, positive environment. ${ }^{29,35,38}$

\section{What are the Elements of Person- Centered Dementia Care?}

Based on the definition above, elements of person-centered dementia care were identified (Figure 2). They were organized into three categories: organizational structure, dementia care process and care outcome.

\section{Organizational Structure}

Supportive and functional management is needed for person-centered dementia care. ${ }^{11,28,29,42}$ Ericson-Lidman et al found that management support and confirmation of caregivers' work and efforts will build a strong care environment. ${ }^{28}$ Their study also found that it was important for management to set a clear value base that permeates all aspects of care. Without a value base, quality of 


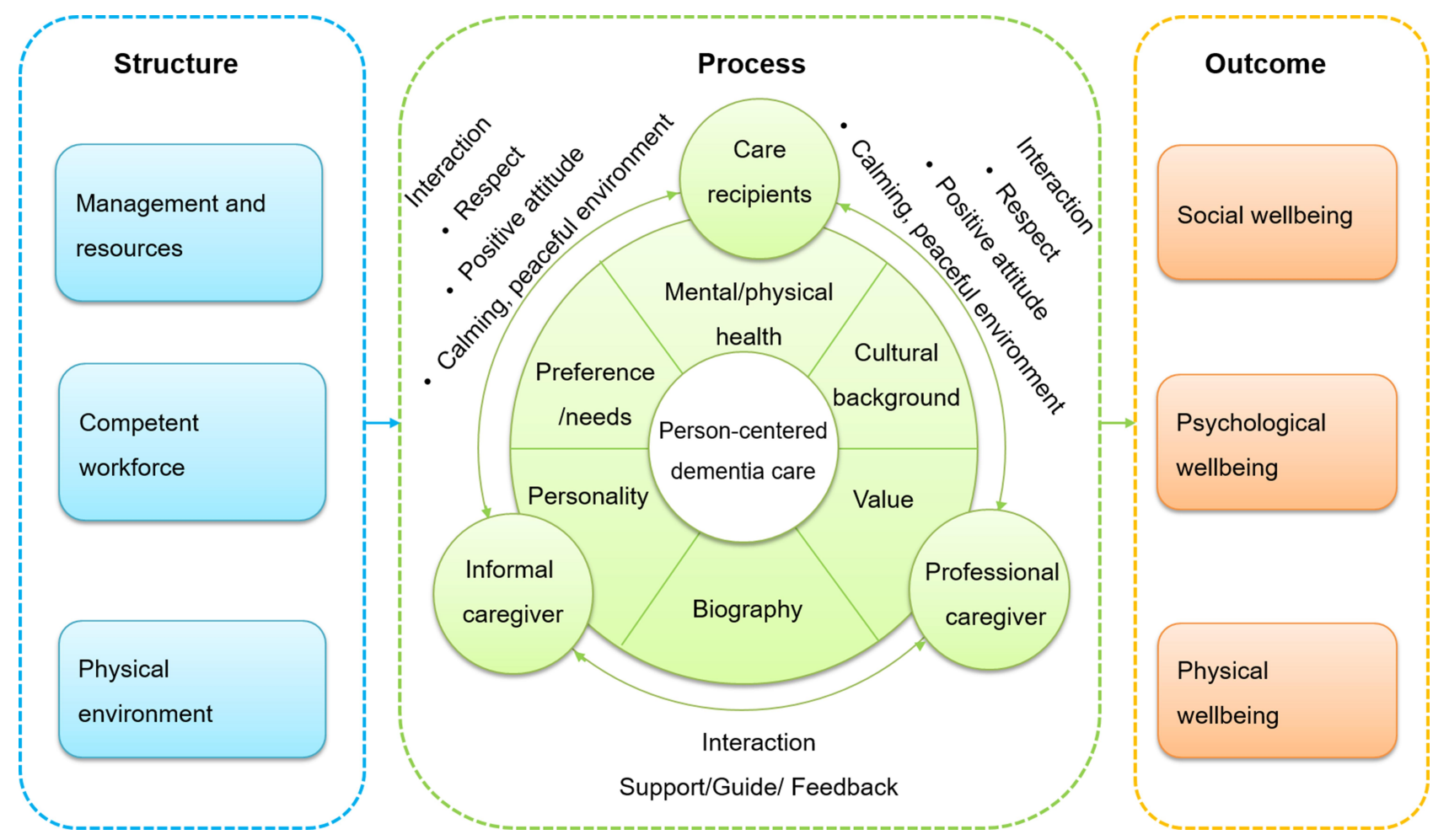

Figure 2 Elements of person-centered dementia care.

care may be jeopardized and caregivers' well-being and job satisfaction may be harmed. Both Scerri et al and Strandroos and Antelius identified the importance of management maintaining the continuity of interpersonal relations between the caregivers and care recipients. ${ }^{29,39}$ This included less staff rotation and less use of substitute caregivers from contracting agencies, as it took time for the caregivers and care recipients to become familiar and "get to know each other". ${ }^{39}$ To do this, adequate resources are needed. $^{37}$

A competent workforce is another critical factor in person-centered dementia care. ${ }^{27-29,32,33}$ It includes education and training experience. Pulsford et al found that older, better educated and more experienced caregivers were more likely to embrace the person-centered approach. ${ }^{27}$ Education is important for caregivers to feel engaged in work and respond to care recipients appropriately during care provision. ${ }^{28}$ Therefore, it will contribute to proper provision of person-centered dementia care. ${ }^{28}$ To achieve better outcomes in education and training, it is important for caregivers to have both opportunity and time for reflection. ${ }^{32}$

A satisfying physical environment is essential for both caregivers and care recipients. ${ }^{27-29,39}$ Good location, supportive architecture and planning and easy access to attractive scenery and nature make a good work environment for caregivers. ${ }^{28}$ A homely, safe, clean and relaxed environment with dementia-friendly design such as colorcoded signs near bathrooms ${ }^{29}$ can help care recipients to use these clues "to make meaning of the place", reducing uncertainty experienced by care recipients. ${ }^{39}$ A good physical environment can also facilitate the interaction between caregivers and care recipients by making the care recipients less aggressive and establishing a common ground between the two parties. ${ }^{27,39}$

\section{Dementia Care Process}

To provide person-centered dementia care, caregivers need to have a good knowledge of care recipients. ${ }^{38}$ This knowledge includes mental and physical health, cultural background, value, biography, personality and preferences and needs. ${ }^{11,28,37,38,40,42}$ In addition to health records, this knowledge may also be obtained through reminiscing with care recipients, ${ }^{43}$ knowledge sharing from other caregivers $^{38}$ and communication with relatives. ${ }^{29,44,45}$ Reminiscing with care recipients can help caregivers to understand who the person is, what matters to them and why they have certain behaviors. ${ }^{32,43}$ It also helps to build up relationships between caregivers and care recipients, leading to a better interaction during care provision. ${ }^{43}$ 
The process of providing dementia care consists of multiple interactions between caregivers and care recipients. ${ }^{30,38,44}$ In the process of interaction, caregivers should respect care recipients, ${ }^{28,30,31,37}$ have a positive attitude $^{33,37}$ and build a calming, peaceful, positive environment. ${ }^{35,38}$

\section{Care Outcome}

Meaningful interaction can promote the social well-being of care recipients. ${ }^{31}$ The word "meaningful" to care recipients signifies being respected and heard, ${ }^{30}$ feeling secure ${ }^{31}$ and receiving a positive attitude from caregivers. ${ }^{33}$ Good interaction also leads to psychological well-being, ${ }^{32,33}$ helping care recipients to stay calm and peaceful. ${ }^{38}$ This consequently results in cooperation from care recipients in smooth care delivery and improved physical well-being. ${ }^{27,28}$

\section{How Do Caregivers Interact with Care Recipients with Dementia?}

Six purposes and approaches adopted by caregivers to achieve these purposes were identified from the literature (Figure 3).

\section{Know and Understand Care Recipients}

Reminiscing with care recipients was mentioned as an approach to know and understand care recipients. ${ }^{30,32,35,37,40,43,45}$ This can be done using artefacts such as photos to trigger conversations. ${ }^{40,45}$ Cooney et al specifically studied this approach. ${ }^{43}$ They found that it helped caregivers to know about care recipients beneath the dementia through their memories, thus enabling the caregivers to understand care recipients and their behaviors better. This eventually changed how the caregivers interacted with care recipients with dementia.

In addition, caregivers read care recipients' body language such as pushing away a spoon or open mouth for food alongside facial expressions to understand them. ${ }^{39}$ To do this, the caregivers needed to have a long, continuous relationship with the care recipients. ${ }^{39}$

\section{Keep Care Recipients Happy and Satisfied} Four approaches were used to keep care recipients happy and satisfied. These approaches included caregivers showing their own good feelings such as happiness and satisfaction to care recipients, ${ }^{28}$ facilitating, showing interest in and encouraging care recipients to do things they enjoy, ${ }^{29,39,46,47}$ laughing together, ${ }^{30}$ and noticing, complimenting and celebrating efforts of care recipients. ${ }^{29,35}$

\section{Make Care Recipients Feel Safe and Secure}

Three approaches were mentioned in the literature about how to make care recipients feel safe and secure. The first approach was to keep a daily care routine. ${ }^{28}$ The second approach was to use body language, gesture, voice tone and facial expression that care recipients feel comfortable with, ${ }^{38,39} \mathrm{eg}$, gently pat the person with a smile. ${ }^{39}$ The third approach was used in a group activity. When a group of care recipients were doing the same activity, it was important that a timeframe was set and to make sure that care recipients completed the activity at about the same time. ${ }^{38}$ Falling behind may make some care recipients anxious.

\section{Calm Care Recipients and Maintain a Peaceful Environment}

Nine approaches were used by caregivers to calm care recipients who were already worried, anxious, stressed, jealous, angry or offensive. The first approach was to go along with care recipients to reassure them, ${ }^{29,35,47-49} \mathrm{eg}$ if a care recipient was worried about no money to pay for the room and food, a caregiver could tell the person that things had been paid for already. ${ }^{35}$ The second approach was to distract care recipients, ${ }^{28,35,37,40}$ eg if a care recipient kept worrying about something, a caregiver could distract the person by providing markers and paper to draw, ${ }^{35}$ talk about different topics ${ }^{37}$ or just simply sit down with the person alone. ${ }^{37}$ The third approach was to get a different caregiver to deal with the troublesome situation immediately. ${ }^{28}$

The fourth approach was to minimize or remove the stimuli or whatever caused the trouble. ${ }^{28}$ For example, closing a door to minimize sound from other activities and people, ${ }^{38}$ not conducting two care activities at the same time ${ }^{38}$ and asking relatives not to visit during mealtime because some care recipients may become anxious when seeing an unknown face. ${ }^{28}$ The fifth approach was to confront, ignore or provoke in order to deal with challenging behaviors. ${ }^{32}$ The sixth approach was to praise or say something positive to care recipients who were resistant or exhibited challenging behaviors. ${ }^{32,48}$

The seventh approach was to help care recipients to focus on the task at hand by being together with them and 


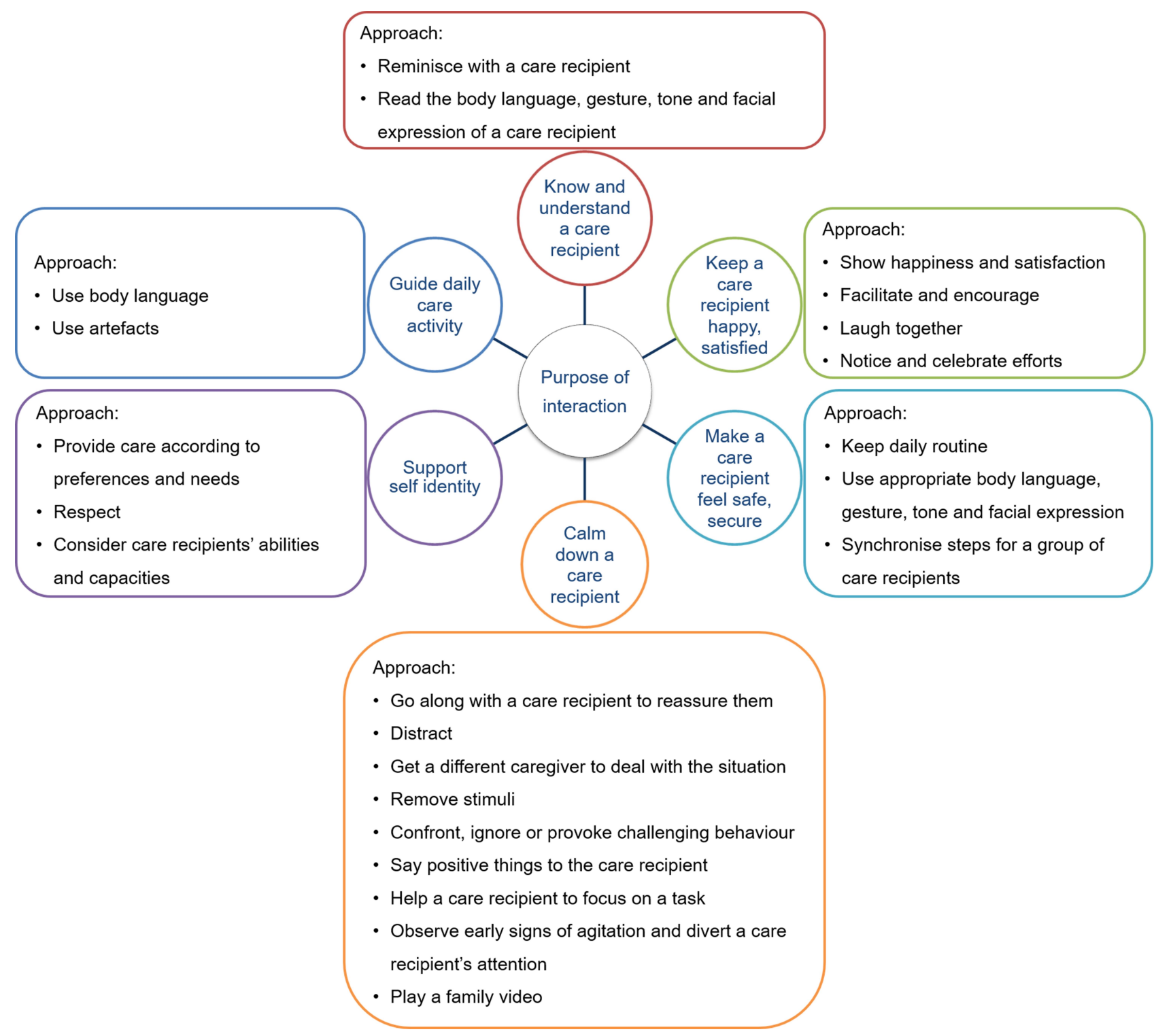

Figure 3 Interaction approaches adopted by caregivers in person-centered dementia care.

continuously encouraging them. ${ }^{38}$ The eighth was to observe early signs of agitation and divert a care recipient's attention immediately before the person became agitated, eg, changing the topic of conversation or using humor. $^{38}$ The ninth was to play a family video to ease anxiety and anger. ${ }^{49}$ Although these approaches were numbered in order to describe them, there was no specific order when applying them in practice.

\section{Support Self-Identity of Care Recipients}

Three approaches were identified from the literature. The first one was to provide care according to care recipients' preferences and needs, ${ }^{11,28,38,40,47,50}$ eg choosing food that the person preferred and could eat without much difficulty. ${ }^{38}$ The second approach was to respect care recipients, eg, knocking on the door before entering and not talking over the head of a care recipient to a co-worker when fulfilling the person's needs. ${ }^{28}$ The third approach was to consider the care recipients' abilities and capacities. ${ }^{28,42}$ This requires the caregivers neither to do things for care recipients which they could still do themselves, ${ }^{42}$ nor to demand them to do things exceeded their capacities, eg, asking several questions or giving several options at a time. ${ }^{28}$ It also requires the caregivers to adjust to the pace of the person. $^{46}$ 


\section{Guide Care Recipients to Conduct Daily Care Activities}

In situations where caregivers and care recipients did not share a common spoken language, caregivers used body language and artefacts to guide care recipients to conduct basic care activities such as eating and drinking. ${ }^{39,46}$ For example, to suggest the person watch TV, the caregiver walked the person to the TV room. ${ }^{39}$ Similar patterns were also found with other artefacts such as drinking glasses, toothbrushes and combs of which the person had previous experience.

\section{Discussion}

This review addressed the gap in the literature that there is a lack of synthesis of recent evidence on what interaction approaches are used by caregivers according to the principles of person-centered dementia care. The contributions of this review include discussion of the definition of person-centered dementia care, identification of elements of person-centered dementia care and identification of interaction approaches adopted by caregivers.

The organizational structure - management and resources, nursing competency and the physical environment - provides the foundation for person-centered dementia care. A supportive management that sets a clear value base and ensures the continuity of care is essential for a strong care environment to deliver person-centered dementia care. ${ }^{28}$ However, the scarcity of resources available is a constraint on delivering person-centered dementia care, as research has shown that when resources are scarce, caregivers seem to focus more on the physiological needs. ${ }^{51}$ It may suggest that caregivers feel time pressure which might generate a troubled conscience, ${ }^{28}$ eg guilt for not spending enough time with care recipients. This troubled conscience may become another obstacle for caregivers to deliver person-centered care which requires the person to be calm, patient, happy and kind. ${ }^{39}$

Another critical component of the organizational structure is a competent workforce which has always been a challenge for residential aged care homes to recruit and retain. ${ }^{52}$ Although lack of job satisfaction has been found to be the main reason for caregivers to quit the dementia care labor market, ${ }^{53}$ a systematic literature review found that the intrinsic rewards of caring for people with dementia actually attracted caregivers. ${ }^{52}$ It also found that essential strategies for retention include ongoing education and training for skills, leadership and teamwork were among the essential strategies for their retention. ${ }^{52}$

This review found that a satisfying physical environment is a critical facilitator for person-centered dementia care. Design features such as tidy, organized environment and careful use of colors and color contrast could be helpful for people with dementia. ${ }^{54}$ These features could be used as general principles but adapted for individuals to make them person-centered. Improved physical environment according to the principles of person-centered environment is found to have positive impacts on quality of life and agitation for people with dementia. ${ }^{55}$

As the actual process of delivering person-centered dementia care is through interactions between caregivers and care recipients with dementia, there are arguments to shift from person-centered care to relationship-centered care (eg, the Senses Framework ${ }^{10,56}$ ) as the latter is more practical to implement. ${ }^{11}$ Despite its importance, relationship-centered care does not address wider aspects of the workplace $^{41}$ such as management and the physical environment which influences care practices. ${ }^{28,29}$

Three outcomes of person-centered dementia care emerged from the literature: social well-being, psychological well-being and physical well-being. Social wellbeing is found to be related to caregivers' attitudes, an element identified for interaction between caregivers and care recipients with dementia in this literature review (Figure 2). Caregivers' positive attitudes are associated with care recipients' better social well-being, as found in a recent cross-sectional questionnaire survey study conducted with 291 caregivers in 15 residential aged care homes in Netherlands. ${ }^{57}$ Psychological well-being of care recipients with dementia can be improved by person-centered care, as demonstrated by a randomized controlled trial conducted in 38 Australian residential aged care homes. ${ }^{55,58}$ Physical well-being is also shown to be associated with person-centered dementia care in a Swedish national survey study. ${ }^{59}$ While these associations are confirmed, further empirical research is needed to understand how the overall well-being is associated with person-centered dementia care. This will require development of a comprehensive list of quantifiable indicators of well-being of care recipients.

This review identified interaction approaches used by caregivers when providing person-centered dementia care. Good interactions with a care recipient with dementia can enhance the well-being of the person, especially when the interactions support the person's self-identity. ${ }^{34}$ Many 
studies articulated the use of the reminiscing approach by caregivers to know and understand care recipients, suggesting a wide adoption of this approach in the delivery of person-centered dementia care. The number of approaches used for the purpose of calming down a care recipient is much more than that for the other purposes, indicating the complex situations that caregivers need to address when delivering the care. One of the approaches used by caregivers to keep a care recipient happy and satisfied is to show their happiness and satisfaction to the person. As this approach can lead to successful interactions with care recipients with dementia, ${ }^{28}$ it is important for caregivers to be aware of and regulate their mood.

Research shows that organisational culture can affect the person-centered care experience of people with dementia. ${ }^{60,61}$ Although organisational culture is not explicitly identified in Figure 2 as an element of person-centered dementia care, it is manifested as values ${ }^{62}$ that are set by the management as part of the organisational structure and permeate all aspects of care through the dementia care process.

\section{Implications for Practice}

Providing quality person-centered dementia care requires managers of residential aged care homes to consider from three important aspects - structure, process and outcome. The managers could use Figure 2 as a roadmap to evaluate current dementia care practice, to develop a plan to implement person-centered dementia care or to identify areas for improvement.

The interaction approaches identified in this review may assist caregivers in care provision. In order to choose the most appropriate approach for a particular situation, the caregivers need to be sensitive to the care recipients' experiences $^{63}$ and be able to read subtle signs and bodily expressions of the care recipients. ${ }^{42}$ Specific training on how to interact with care recipients in both spoken and body language will be useful for caregivers to deliver effective interactions.

\section{Limitations}

Only articles published between January 2000 and June 2020 were searched. The studies included in this review were almost exclusively conducted in European countries, limiting the understanding of the status of person-centered dementia care in other countries, especially the lowincome and middle-income countries where two-thirds of the dementia population reside. ${ }^{1,64}$ A more comprehensive review method could be used in future research to identify studies from these countries.

\section{Conclusions}

Interaction between caregivers and care recipients with dementia plays an important role in providing personcentered dementia care. This review identified interaction approaches used by caregivers when delivering this care. It also identified the elements of person-centered dementia care. The findings of this review can be used to guide the implementation, evaluation and improvement effort of person-centered dementia care and to provide training for caregivers to improve their interactions with care recipients with dementia.

All the studies were conducted in high-income countries and almost all of them were conducted in Europe. As $58 \%$ of the world's dementia population were distributed in low-income and middle-income countries and the number is still at a relatively high rate of growth, ${ }^{65}$ it is paramount for future research to investigate the status of person-centered dementia care in these countries in which the social structure, cultural values, ethnicity and health care system, etc., are different from high-income countries.

\section{Data Sharing Statement}

Data generated or analyzed during this study are included in this published article and its supplementary information files.

\section{Author Contributions}

All authors made substantial contributions to conception and design, acquisition of data, or analysis and interpretation of data; took part in drafting the article or revising it critically for important intellectual content; gave final approval of the version to be published; and agree to be accountable for all aspects of the work.

\section{Funding}

The authors would like to thank the Clinical Key Specialty of Guangzhou Medical University and the following projects for their financial support: the Medical Science and Technology Research Foundation of Guangdong Province (No. A2019466); the Guangzhou Health Science and Technology General Guidance Project (No. 20191A011090); the Key Technology Research Project of Guangzhou Science, Technology and Innovation Committee (No. 201902020001); and the Science and Technology Project of Guangzhou (No. 201905010004). 


\section{Disclosure}

The authors report no conflicts of interest for this work.

\section{References}

1. Alzheimer's Disease International. World Alzheimer Report 2019: Attitudes to Dementia. London: Alzheimer's Disease International; 2019.

2. World Health Organization. Dementia: A Public Health Priority. World Health Organization; 2012.

3. Kitwood T. The technical, the personal, and the framing of dementia. Soc Behav. 1988.

4. Brooker D. What is person-centred care in dementia? Rev Clin Gerontol. 2003;13(3):215-222. doi:10.1017/S095925980400108X

5. Fazio S, Pace D, Flinner J, Kallmyer B. The fundamentals of personcentered care for individuals with dementia. Gerontologist. 2018;58 (suppl_1):S10-S19. doi:10.1093/geront/gnx122

6. Kitwood TM. Dementia Reconsidered: The Person Comes First. Open university press; 1997.

7. Brooker D, Latham I. Person-Centred Dementia Care: Making Services Better with the VIPS Framework. Jessica Kingsley Publishers; 2015.

8. Edvardsson D, Winblad B, Sandman P-O. Person-centred care of people with severe Alzheimer's disease: current status and ways forward. Lancet Neurol. 2008;7(4):362-367. doi:10.1016/S14744422(08)70063-2

9. Love K, Pinkowitz J. Person-centered care for people with dementia: a theoretical and conceptual framework. Generations. 2013;37(3):23-29.

10. Nolan MR, Davies S, Brown J, Keady J, Nolan J. Beyond 'personcentred'care: a new vision for gerontological nursing. J Clin Nurs. 2004;13:45-53. doi:10.1111/j.1365-2702.2004.00926.x

11. Ryan T, Nolan M, Reid D, Enderby P. Using the senses framework to achieve relationship-centred dementia care services: a case example. Dementia. 2008;7(1):71-93. doi:10.1177/1471301207085368

12. de Witt L, Fortune D. Relationship-centered dementia care: insights from a community-based culture change coalition. Dementia. 2019;18(3):1146-1165. doi:10.1177/1471301217708814

13. Adams T, Gardiner P. Communication and interaction within dementia care triads: developing a theory for relationship-centred care. Dementia. 2005;4(2):185-205. doi:10.1177/1471301205051092

14. McCormack B. Person-centredness in gerontological nursing: an overview of the literature. J Clin Nurs. 2004;13:31-38. doi:10.1111/ j.1365-2702.2004.00924.x

15. Featherstone RM, Dryden DM, Foisy M, et al. Advancing knowledge of rapid reviews: an analysis of results, conclusions and recommendations from published review articles examining rapid reviews. Syst Rev. 2015;4(1):50. doi:10.1186/s13643-015-0040-4

16. Luckett T, Phillips J, Agar M, Virdun C, Green A, Davidson PM. Elements of effective palliative care models: a rapid review. $B M C$ Health Serv Res. 2014;14(1):136. doi:10.1186/1472-6963-1 4-136

17. Lal S, Adair CE. E-mental health: a rapid review of the literature. Psychiatr Serv. 2014;65(1):24-32. doi:10.1176/appi.ps.201300009

18. Lafortune L, Martin S, Kelly S, et al. Behavioural risk factors in midlife associated with successful ageing, disability, dementia and frailty in later life: a rapid systematic review. PLoS One. 2016;11(2): e0144405. doi:10.1371/journal.pone. 0144405

19. Hartling L, Guise J-M, Hempel S, et al. EPC Methods: AHRQ EndUser Perspectives of Rapid Reviews. Agency for Healthcare Research and Quality (US); 2016.

20. Watt A, Cameron A, Sturm L, et al. Rapid reviews versus full systematic reviews: an inventory of current methods and practice in health technology assessment. Int J Technol Assess Health Care. 2008;24(2):133-139. doi:10.1017/S0266462308080185
21. Ganann R, Ciliska D, Thomas H. Expediting systematic reviews: methods and implications of rapid reviews. Implement Sci. 2010;5 (1):56. doi:10.1186/1748-5908-5-56

22. Thomas J, Harden A. Methods for the thematic synthesis of qualitative research in systematic reviews. BMC Med Res Methodol. 2008;8 (1):45. doi:10.1186/1471-2288-8-45

23. Donabedian A. The quality of care: how can it be assessed? JAMA. 1988;260(12):1743-1748. doi:10.1001/jama.1988.03410120089033

24. McGilton KS, Heath $\mathrm{H}$, Chu $\mathrm{CH}$, et al. Moving the agenda forward: a person-centred framework in long-term care. Int $J$ Older People Nurs. 2012;7(4):303-309. doi:10.1111/opn.12010

25. Santana MJ, Manalili K, Jolley RJ, Zelinsky S, Quan H, Lu M. How to practice person-centred care: a conceptual framework. Health Expect. 2018;21(2):429-440. doi:10.1111/hex.12640

26. Amador S, Sampson EL, Goodman C, Robinson L, Team SR. A systematic review and critical appraisal of quality indicators to assess optimal palliative care for older people with dementia. Palliat Med. 2019;33(4):415-429. doi:10.1177/0269216319834227

27. Pulsford D, Duxbury JA, Hadi M. A survey of staff attitudes and responses to people with dementia who are aggressive in residential care settings. J Psychiatr Ment Health Nurs. 2011;18(2):97-104. doi:10.1111/j.1365-2850.2010.01646.x

28. Ericson-Lidman E, Larsson L-L, Norberg A. Caring for people with dementia disease (DD) and working in a private not-for-profit residential care facility for people with DD. Scand J Caring Sci. 2014;28 (2):337-346. doi:10.1111/scs.12063

29. Scerri A, Innes A, Scerri C. Discovering what works well: exploring quality dementia care in hospital wards using an appreciative inquiry approach. J Clin Nurs. 2015;24(13-14):1916-1925. doi:10.1111/ jocn. 12822

30. Alsawy S, Tai S, McEvoy P, Mansell W. 'It's nice to think somebody's listening to me instead of saying "oh shut up". People with dementia reflect on what makes communication good and meaningful. $J$ Psychiatr Ment Health Nurs. 2020;27(2):151-161. doi:10.1111/jpm.12559

31. Brataas HV, Bjugan H, Wille T, Hellzen O. Experiences of day care and collaboration among people with mild dementia. J Clin Nurs. 2010;19(19-20):2839-2848. doi:10.1111/j.1365-2702.2010.03270.x

32. Graneheim UH, Jansson L, Lindgren B-M. Hovering between heaven and hell: an observational study focusing on the interactions between one woman with schizophrenia, dementia, and challenging behaviour and her care providers. Issues Ment Health Nurs. 2015;36(7):543550. doi:10.3109/01612840.2015.1007540

33. Norbergh K-G, Helin Y, Dahl A, Hellzén O, Asplund K. Nurses' attitudes towards people with dementia: the semantic differential technique. Nurs Ethics. 2006;13(3):264-274. doi:10.1191/0969733006ne863oa

34. Willemse BM, Downs M, Arnold L, Smit D, de Lange J, Pot AM. Staff-resident interactions in long-term care for people with dementia: the role of meeting psychological needs in achieving residents' well-being. Aging Ment Health. 2015;19(5):444-452. doi:10.1080/ 13607863.2014.944088

35. Teitelman J, Raber C, Watts J. The power of the social environment in motivating persons with dementia to engage in occupation: qualitative findings. Phys Occup Ther Geriatr. 2010;28(4):321-333. doi:10.3109/02703181.2010.532582

36. Jeon Y-H, Luscombe G, Chenoweth L, et al. Staff outcomes from the caring for aged dementia care resident study (CADRES): a cluster randomised trial. Int J Nurs Stud. 2012;49(5):508-518. doi:10.1016/j. ijnurstu.2011.10.020

37. Heggestad AKT, Nortvedt P, Slettebø Å. Dignity and care for people with dementia living in nursing homes. Dementia (London, England). 2015;14(6):825-841. doi:10.1177/1471301213512840

38. Bergland Å, Johansen H, Sellevold GS. A qualitative study of professional caregivers' perceptions of processes contributing to mealtime agitation in persons with dementia in nursing home wards and strategies to attain calmness. Nurs Open. 2015;2(3):119-129. doi:10.1002/nop2.24 
39. Strandroos L, Antelius E. Interaction and common ground in dementia: communication across linguistic and cultural diversity in a residential dementia care setting. Health (London). 2017;21(5):538-554. doi:10.1177/1363459316677626

40. Wilson CB, Davies S. Developing relationships in long term care environments: the contribution of staff. J Clin Nurs. 2009;18 (12):1746-1755. doi:10.1111/j.1365-2702.2008.02748.x

41. Snoeren MMWC, Janssen BM, Niessen TJH, Abma TA. Nurturing cultural change in care for older people: seeing the cherry tree blossom. Health Care Anal. 2014;24(4):349-373.

42. Watson J. Developing the senses framework to support relationshipcentred care for people with advanced dementia until the end of life in care homes. Dementia (London, England). 2016;18(2):545-566.

43. Cooney A, Hunter A, Murphy K, et al. 'Seeing me through my memories': a grounded theory study on using reminiscence with people with dementia living in long-term care. J Clin Nurs. 2014;23(23-24):3564-3574. doi:10.1111/jocn.12645

44. de Rooij AHPM, Luijkx KG, Spruytte N, Emmerink PMJ, Schols JMGA, Declercq AG. Family caregiver perspectives on social relations of elderly residents with dementia in small-scale versus traditional long-term care settings in the Netherlands and Belgium. J Clin Nurs. 2012;21(2122):3106-3116. doi:10.1111/j.1365-2702.2012.04110.x

45. Kelley R, Godfrey M, Young J. The impacts of family involvement on general hospital care experiences for people living with dementia: an ethnographic study. Int J Nurs Stud. 2019;96:72-81. doi:10.1016/j. ijnurstu.2019.04.004

46. Gilmore-Bykovskyi AL. Caregiver person-centeredness and behavioral symptoms during mealtime interactions: development and feasibility of a coding scheme. Geriatr Nurs (Minneap). 2015;36(2): S10-S15. doi:10.1016/j.gerinurse.2015.02.018

47. Savundranayagam MY. Missed opportunities for person-centered communication: implications for staff-resident interactions in longterm care. Int Psychogeriatr. 2014;26(4):645-655. doi:10.1017/ S1041610213002093

48. Plejert C, Jansson G, Yazdanpanah M. Response practices in multilingual interaction with an older Persian woman in a Swedish residential home. J Cross Cult Gerontol. 2014;29(1):1-23. doi:10.1007/ s10823-013-9217-2

49. Hung L, Au-Yeung A, Helmer C, et al. Feasibility and acceptability of an iPad intervention to support dementia care in the hospital setting. Contemp Nurse. 2018;54(4-5):350-361. doi:10.1080/103761 78.2018.1505436

50. Jaycock S, Persaud M, Johnson R. The effectiveness of dementia care mapping in intellectual disability residential services: a follow-up study. J Intellect Disabil. 2006;10(4):365-375. doi:10.1177/17446 29506072870

51. Slettebø Å, Kirkevold M, Andersen B, et al. Clinical prioritizations and contextual constraints in nursing homes-a qualitative study. Scand J Caring Sci. 2010;24(3):533-540. doi:10.1111/j.1471-6712. 2009.00745.x
52. Chenoweth L, Jeon YH, Merlyn T, Brodaty H. A systematic review of what factors attract and retain nurses in aged and dementia care. J Clin Nurs. 2010;19(1-2):156-167. doi:10.1111/j.1365-2702.2009.02955.x

53. Vernooij-Dasssen MJ, Faber MJ, Olde Rikkert MG, et al. Dementia care and labour market: the role of job satisfaction. Aging Ment Health. 2009;13(3):383-390. doi:10.1080/13607860902861043

54. Hung L, Phinney A, Chaudhury H, Rodney P, Tabamo J, Bohl D. 'Little things matter!' Exploring the perspectives of patients with dementia about the hospital environment. Int J Older People Nurs. 2017;12(3):n/a-n/a. doi:10.1111/opn.12153

55. Chenoweth L, Forbes I, Fleming R, et al. PerCEN: a cluster randomized controlled trial of person-centered residential care and environment for people with dementia. International Psychogeriatrics. 2014;26(7):1147-1160. doi:10.1017/S1041610214000398

56. Nolan M, Davies S, Ryan T, Keady J. Relationship-centred care and the 'Senses' framework. J Dementia Care. 2008;16(1):26-28.

57. Gerritsen D, van Beek A, Woods R. Relationship of care staff attitudes with social well-being and challenging behavior of nursing home residents with dementia: a cross sectional study. Aging Ment Health. 2019;23 (11):1517-1523. doi:10.1080/13607863.2018.1506737

58. Chenoweth L, Jeon Y-H, Stein-Parbury J, et al. PerCEN trial participant perspectives on the implementation and outcomes of personcentered dementia care and environments. Int Psychogeriatr. 2015;27 (12):2045. doi:10.1017/S1041610215001350

59. Sjögren K, Lindkvist M, Sandman P-O, Zingmark K, Edvardsson D. Person-centredness and its association with resident well-being in dementia care units. J Adv Nurs. 2013;69(10):2196-2205. doi:10.11 11/jan. 12085

60. Killett A, Burns D, Kelly F, et al. Digging deep: how organisational culture affects care home residents' experiences. Ageing Soc. 2016;36 (1):160-188. doi:10.1017/S0144686X14001111

61. Kirkley C, Bamford C, Poole M, Arksey H, Hughes J, Bond J. The impact of organisational culture on the delivery of person-centred care in services providing respite care and short breaks for people with dementia. Health Soc Care Community. 2011;19(4):438-448. doi:10.1111/j.1365-2524.2011.00998.x

62. Schein EH. Organizational Culture. Vol. 45. American Psychological Association; 1990.

63. Hartrick G. Relational capacity: the foundation for interpersonal nursing practice. J Adv Nurs. 1997;26(3):523-528. doi:10.1046/ j.1365-2648.1997.t01-12-00999.x

64. Rodriguez JJL, Ferri CP, Acosta D, et al. Prevalence of dementia in Latin America, India, and China: a population-based cross-sectional survey. Lancet. 2008;372(9637):464-474. doi:10.1016/S0140-6736 (08)61002-8

65. Prince M, Wimo A, Guerchet M, Ali G, Wu Y, Prina M. World Alzheimer Report 2015. The Global Impact of Dementia. Alzheimer's Disease International. London: Alzheimer's Disease International (ADI); 2015.
Clinical Interventions in Aging

\section{Publish your work in this journal}

Clinical Interventions in Aging is an international, peer-reviewed journal focusing on evidence-based reports on the value or lack thereof of treatments intended to prevent or delay the onset of maladaptive correlates of aging in human beings. This journal is indexed on PubMed Central, MedLine, CAS, Scopus and the Elsevier
Bibliographic databases. The manuscript management system is completely online and includes a very quick and fair peer-review system, which is all easy to use. Visit http://www.dovepress.com/ testimonials.php to read real quotes from published authors. 